\title{
Cerebrospinal Fluid Levels of Monoamine Metabolites
}

\section{A Preliminary Study of Their Relation to Menstrual Cycle Phase, Sex Steroids, and Pituitary Hormones in Healthy Women and in Women with Premenstrual Syndrome}

Elias Eriksson, M.D., Ph.D., Christer Alling, M.D., Ph.D., Björn Andersch, M.D., Ph.D., Kerstin Andersson, M.D., Ph.D., and Ulf Berggren, M.D., Ph.D.

The cerebrospinal fluid (CSF) levels of the serotonin metabolite 5-hydroxyindoleacetic acid (5-HIAA), the noradrenaline metabolite 3-methoxy-4-hydroxyphenylethylene glycol (MHPG), and the dopamine metabolite homovanillic acid (HVA) were measured in a group of drug-free non-depressed women with premenstrual syndrome (PMS) (late luteal phase dysphoric disorder) $(\mathrm{n}=13)$ and in controls with no premenstrual complaints $(\mathbf{n}=13)$. In six patients and eight controls, CSF samples from both the luteal and the follicular phase were obtained, whereas in the remainder of the subjects, samples from either the follicular phase (patients: 4, controls: 2) or the luteal phase (patients: 3, controls: 3) were taken. The following observations were made: (1) Neither in the follicular phase nor in the luteal phase did the mean concentrations of CSF monoamine metabolites in the PMS group differ from the corresponding values in the control group. (2) Neither in the PMS group nor in the control group did the mean concentrations of monoamine metabolites in CSF samples obtained in the luteal phase differ from the corresponding values obtained in the follicular phase. (3) The intraindividual, intersample variations of CSF HVA and 5-HIAA concentrations were significantly smaller in the PMS group than in the control group. (4) CSF HVA correlated strongly to CSF 5-HIAA in the luteal phase of both patients and controls whereas in the follicular phase, particularly in controls, this correlation was much weaker. (5) In the luteal phase, the CSF HVA/5-HIAA ratio correlated negatively to serum levels of estradiol, progesterone, and testosterone. (6) The CSF HVA/5-HIAA ratio was significantly lower in PMS patients than in controls. (7) A positive correlation between CSF MHPG and serum luteinizing hormone was observed in the follicular phase. (8) A positive correlation between CSF HVA and serum prolactin was observed in the luteal phase. Because the study was comprised of a small number of subjects, the reported findings until replicated should be interpreted with caution.

[Neuropsychopharmacology 11:201-213, 1994]
Received January 10, 1994; revised June 14, 1994; accepted June 20 , 1994.

From the Departments of Pharmacology (EE), Obstetrics/Gynaecology (BA, KA), and Psychiatry/Neurochemistry (UB), University of Göteborg, and Department of Psychiatry/Neurochemistry (CA), University of Lund, Sweden.

Address reprint requests to: Elias Eriksson, Department of Pharmacology, University of Göteborg, Medicinaregatan 7, S-413 90 Göteborg, Sweden.
KEY WORDS: Late luteal phase dysphoric disorder; Premenstrual syndrome (PMS); Cerebrospinal fluid (CSF); 5-hydroxyindoleacetic acid (5-HIAA); 3-methoxy4-hydroxyphenylethylene glycol (MHPG); Homovanillic acid (HVA); Monoamine metabolites; Estradiol;

Progesterone; Testosterone; Luteinizing hormone $(\mathrm{LH})$; Prolactin 
The premenstrual syndrome (PMS), or late luteal phase dysphoric disorder (LLPDD), is characterized by a cluster of symptoms that appear in the luteal phase of most menstrual cycles and which disappear within a few days after the onset of menstruation. Among the mental symptoms, irritability, depressed mood, and carbohydrate craving are some of the most prominent (Frank 1931; Halbreich et al. 1982; American Psychiatric Association 1985).

On the basis of both animal experiments and clinical studies, serotonin is attributed as being important for the regulation of mood, impulse control, and carbohydrate craving (for ref, see Eriksson and Humble, 1991); moreover, serotonin activity has been shown to be influenced by variations in the serum concentrations of sex steroids (see, e.g., Engel et al. 1979; Sietniks et al. 1983; Long et al. 1983; Battaner et al. 1987; Bitar et al. 1991). Consequently, the hypothesis that alterations in serotonergic neurotransmission may be involved in the pathophysiology of PMS is not unfeasible. Support for this assumption has been obtained from recent studies showing that premenstrual complaints can be markedly reduced by serotonin reuptake inhibitors (Eriksson et al. 1990; Rickels et al. 1990; Stone et al. 1991; Menkes et al. 1992; Sundblad et al. 1992; Wood et al. 1992; Sundblad et al. 1993).

Based upon the assumption that the concentrations of a certain monoamine metabolite in the cerebrospinal fluid (CSF) may reflect the turnover of the corresponding transmitter in the brain (Modigh 1975; Scheinin et al. 1986; Stanley et al. 1986), many studies have been undertaken in which CSF levels of the serotonin metabolite 5-hydroxyindoleacetic acid (5-HIAA), the dopamine metabolite homovanillic acid (HVA), and the noradrenaline metabolite 3-methoxy-4-hydroxyphenylethylene glycol (MHPG), have been measured in psychiatric patients and in controls. A negative correlation between aggression and poor impulse control vs CSF 5-HIAA levels is one of the most replicated findings in these types of studies (Brown et al. 1982; Lidberg et al. 1985; Asberg et al. 1986b; Brown and Linnoila 1990); in addition, some workers have found low CSF 5-HIAA concentrations in subgroups of depressed patients (Åsberg et al. 1984; Gibbons and Davis 1984; Westenberg and Verhoeven 1988). The latter finding is, however, still a matter of controversy (for refs, see Gjerris et al. 1988; Reddy et al. 1992).

As a consequence of the strong positive correlation between CSF levels of HVA and 5-HIAA usually observed, the CSF HVA to 5-HIAA ratio has recently attained increased attention as a putative marker of the interactions between dopamine and serotonin in brain (Agren et al. 1986; Roy et al. 1986; Ảgren et al. 1988; Hsiao et al. 1993; Potter and Manji 1993). Interestingly, recent studies indicate that a low HVA to 5-HIAA ratio is a more reliable marker of depression than is a low CSF 5-HIAA (for references see Reddy et al. 1992).

To our knowledge, CSF monoamine metabolites in PMS have previously been investigated in one study only (Parry et al. 1991), in which no healthy controls were included. The present pilot study had three main purposes. First, to investigate whether PMS, like some other conditions characterized by depressed mood and/or impaired impulse control, is associated with abnormal CSF levels of 5-HIAA and/or HVA, or with an abnormal HVA to 5-HIAA ratio. Second, to examine to what extent CSF monoamine metabolite concentrations, in PMS patients as well as in healthy controls, are influenced by menstrual cycle phase. Third, to explore possible relationships between CSF monoamine metabolite concentrations and serum levels of gonadal and pituitary hormones.

\section{MATERIALS AND METHODS}

Women with premenstrual irritability and/or depressed mood were recruited for a drug trial (the results will be presented elsewhere) by means of a newspaper advertisement, followed by a brief telephone interview, and a subsequent, extensive, structured interview. The primary inclusion criteria were severe irritability and/or depressed mood starting regularly around ovulation or during the two weeks preceding the menstrual bleeding and terminating within a few days after the onset of menstruation as reported by the patient during the interview. In addition, the criteria of LLPDD according to DSM-III-R (American Psychiatric Association 1985; Swedish translation), should be fulfilled. The diagnosis of premenstrual syndrome was confirmed by means of daily self-rating during two consecutive menstrual cycles using a visual analogue scale; subjects not presenting at least a $100 \%$ increase in irritability or depressed mood during the five days preceding the menstrual bleeding as compared to the mean rating of cycle days 6 to 10 were not included in the study.

Female controls, of similar age as the patients, were recruited mainly from the staffs and students at the hospital and research unit where the investigations were undertaken. In structured interviews, all controls reported a complete lack of cycle-related changes in mood. That they were free from a premenstrual increase in irritability or depressed mood was also confirmed by one month of daily self-rating.

Totally, 13 patients and 13 controls were included in the study. The mean age of the patients was 39 years (range: 29-45) and the mean age of the controls was 35 years (range: $27-43$ ). In six patients, a CSF sample was obtained both between day 5 and day 8 of the menstrual cycle (day 1 being the first day of menstruation) 
(follicular phase) and within 5 days before the expected onset of menstruation (the late luteal phase) whereas from the other seven patients, a sample was obtained in either the follicular phase $(n=4)$ or in the late luteal phase $(n=3)$. In eight controls, a CSF sample was obtained both in the follicular phase and in the luteal phase whereas from the other five patients, a sample was obtained in either the follicular phase $(n=2)$ or in the late luteal phase $(n=3)$.

The puncture was performed at 8:00 A.M. before any food intake and with the patients in bed in a recumbent position. Twelve $\mathrm{ml}$ of CSF were obtained and divided into 2-ml portions. 5-HIAA, MHPG, and HVA were measured using a mass fragmentographic method as previously described (Gjerris et al. 1987; Swahn et al. 1976). All samples were analyzed in the same run. Precision data: $\mathrm{HVA}: \mathrm{cv}=2.73 \%$; 5 -HIAA: $\mathrm{cv}=7.63 \%$; MHPG: $\mathrm{cV}=6.89 \%$.

A blood sample $(20 \mathrm{ml})$ for determination of serum concentrations of sex steroids and pituitary hormones was obtained from an antecubital vein immediately before the lumbar puncture. Sex steroids, steroid binding proteins, and pituitary hormones were measured using radioimmunoassays provided by Diagnostic Products, Los Angeles, USA (estradiol, progesterone, testosterone, luteinizing hormone $[\mathrm{LH}]$, follicle stimulating hormone $[\mathrm{FSH}]$, and prolactin), or Famos Diagnostica, Finland (cortisol binding globulin [CBG], sex hormone binding globulin [SHBG]). All interassay and intraassay variations were less than $10 \%$.

\section{Statistics}

CSF concentrations of 5-HIAA, HVA, and MHPG, and the HVA to 5-HIAA ratio, were compared using factorial analysis of variance (ANOVA) with cycle phase (luteal phase and follicular phase, respectively) and group (PMS subjects and controls, respectively) as nominal values. The ANOVA was followed by subsequent $t$-tests for comparisons of the diagnostic groups split with respect to cycle phase, and for the cycle phases split with respect to diagnosis.

For comparisons between groups, $p$-values less than .05 were regarded as statistically significant.

Correlation analyses comprised the following parameters: 5-HIAA, HVA, HVA to 5-HIAA ratio, MHPG, estradiol, progesterone, total testosterone, SHBG, CBG, FSH, LH, and prolactin. Two correlation analyses were undertaken; first, the samples were split with respect to menstrual cycle phase, but not with respect to diagnosis; second, the samples were split with respect to menstrual phase as well as to diagnostic group. The correlation analyses were always followed by Fischer's $r$ to $z$ transformation. In order to analyze the differences between groups with respect to a cer- tain correlation, the confidence interval of the $r$-value was calculated.

\section{RESULTS}

\section{CSF Monoamine Metabolite Concentrations}

Mean CSF concentrations of 5-HIAA, HVA, and MHPG in the follicular and luteal phases of women with PMS and with controls, respectively, are shown in Figure 1. Factorial ANOVA revealed no significant effect of either cycle phase (MHPG: $F=0.004, p=.9$; HVA: $F=$ $0.03, p=.9$; 5-HIAA: $F=0.03, p=.9$ ) or the diagnostic group (MHPG: $\mathrm{F}=0.8, p=.4$; HVA: $F=0.9, p=.3$; 5-HIAA: $F=1.7, p=.2$ ) on the concentrations of any of the three monoamine metabolites.

The intraindividual, intersample variations of CSF 5-HIAA, HVA, and MHPG in the subpopulation of individuals from which a CSF sample was obtained both in the follicular phase and the luteal phase are shown in Figure $2 \mathrm{~A}-\mathrm{C}$. The intraindividual correlations were significantly stronger in the PMS group than in the controls for both HVA (PMS: $r=0.86$; controls: $r=0.21$; $p<.05$ ) and 5-HIAA (PMS: $r=0.77$; controls: $r=-0.44$; $p<.001$ ), but not for MHPG (PMS: $r=0.70$; controls: $r=0.24$; NS).

The interindividual variation of CSF HVA concentrations was significantly smaller in the follicular phase of the controls, both when compared to the luteal phase of the controls $(F=6.1, p<.01)$ and when compared to the follicular phase of PMS patients $(F=3.7, p<.05)$. With respect to the other metabolites, neither diagnosis nor cycle phase significantly influenced the withingroup, interindividual variation.

CSF HVA and CSF 5-HIAA correlated significantly

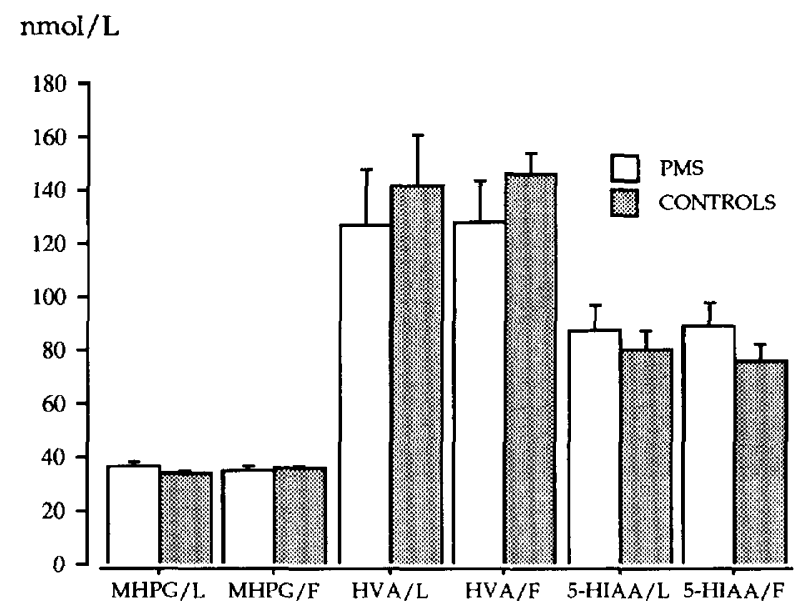

Figure 1. CSF concentrations of MHPG, HVA, and 5-HIAA in the luteal (L) and follicular $(\mathrm{F})$ phases of PMS patients and controls. Bars represent mean \pm SEM. $n=9-11$. For statistics, see Results. 


\section{CONTROLS}

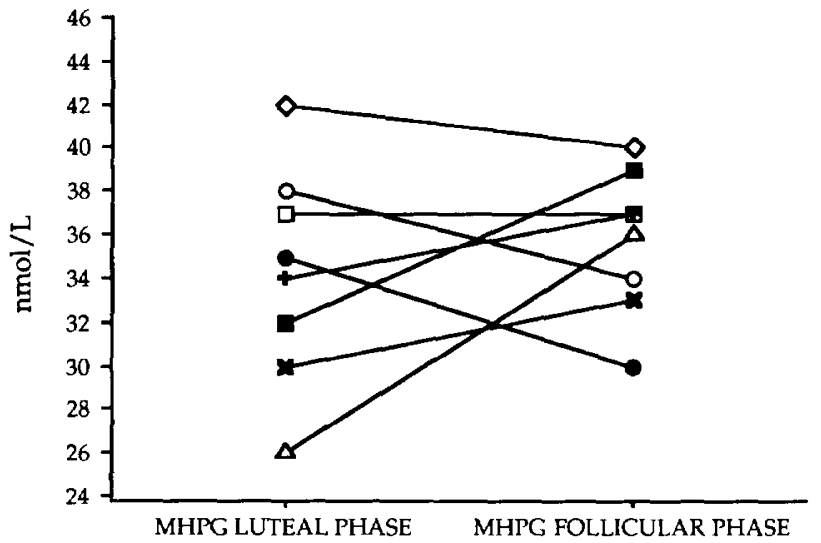

A

\section{CONTROLS}

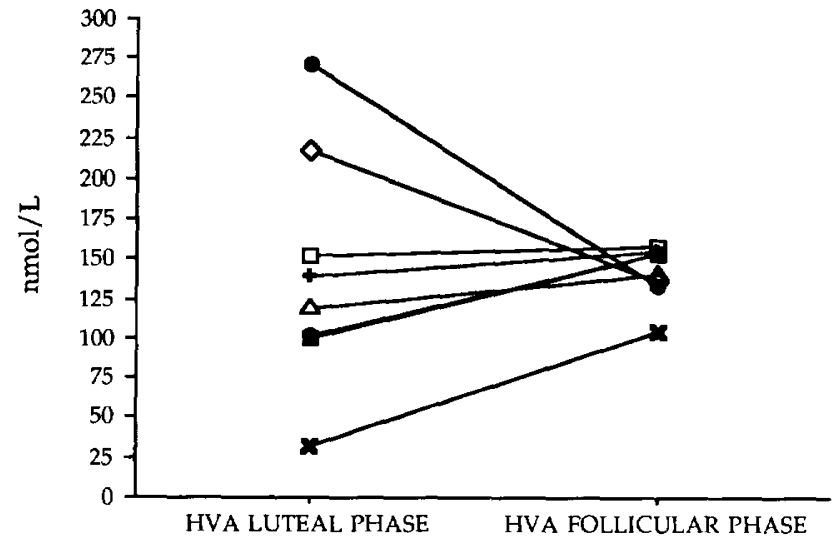

C

\section{CONTROLS}

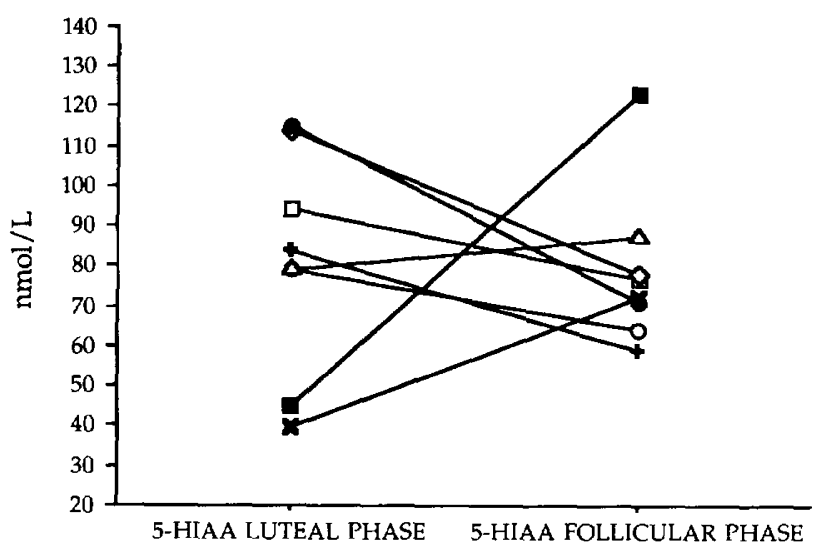

PMS

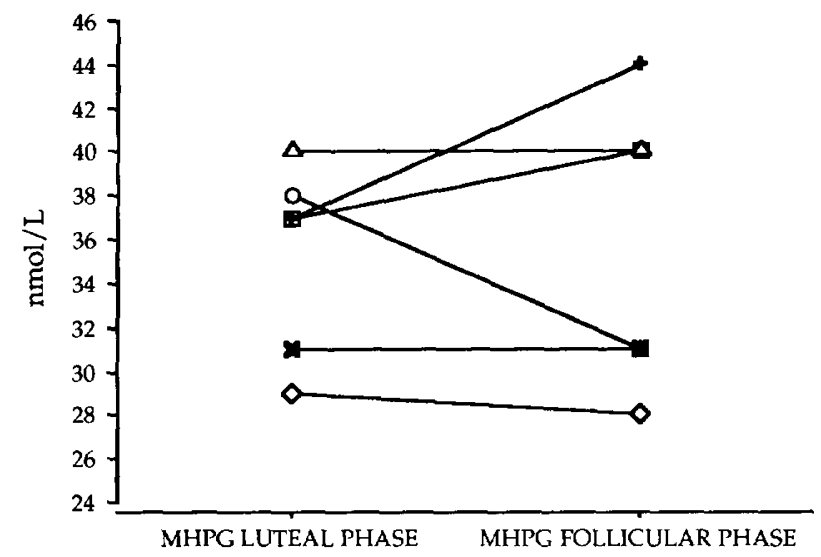

B

\section{PMS}

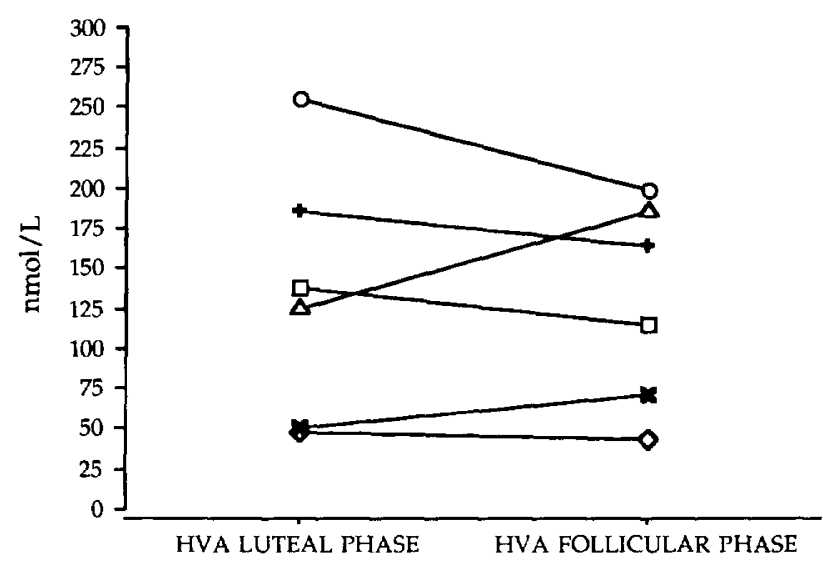

D

\section{PMS}

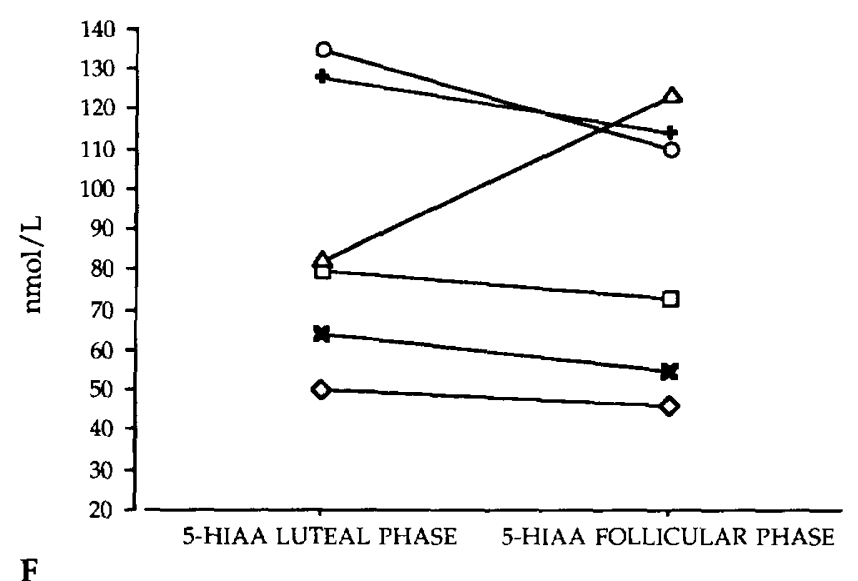

Figure 2. CSF concentrations of MHPG (A-B), HVA (C-D), and 5-HIAA (E-F) in the controls and PMS patients examined in both the luteal phase and the follicular phase. For statistics, see Results.

in the luteal phase of both patients and controls (Figure 3A-B). In contrast, in controls, no correlation between CSF HVA and CSF 5-HIAA was observed in the follicular phase. Also in the PMS group, the HVA versus 5-HIAA correlation appeared weaker in the follicular phase than in the luteal phase; however, the difference in correlation between the follicular and luteal phases, respectively, was statistically significant 


\section{CONTROLS: LUTEAL PHASE}

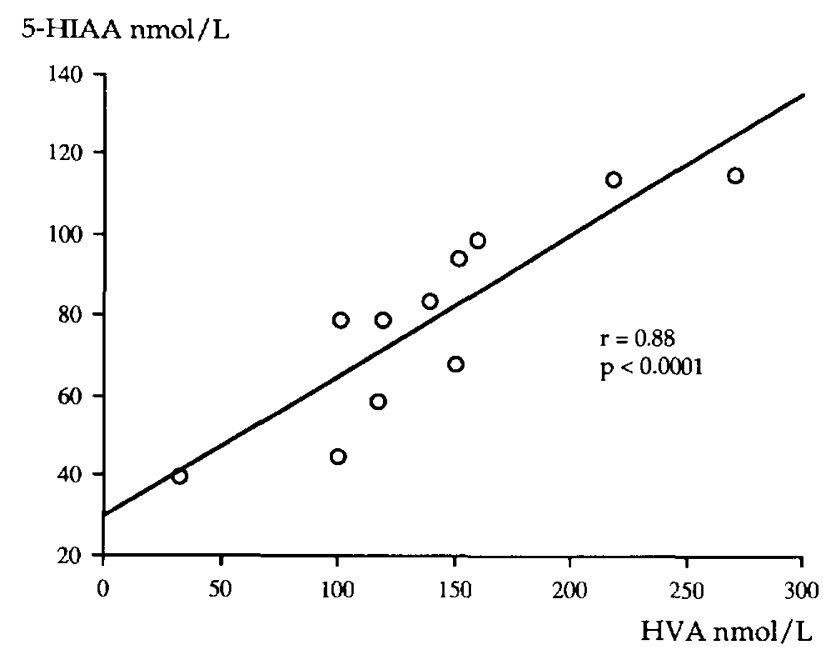

CONTROLS: FOLLICULAR PHASE

5-HIAA nmol/L

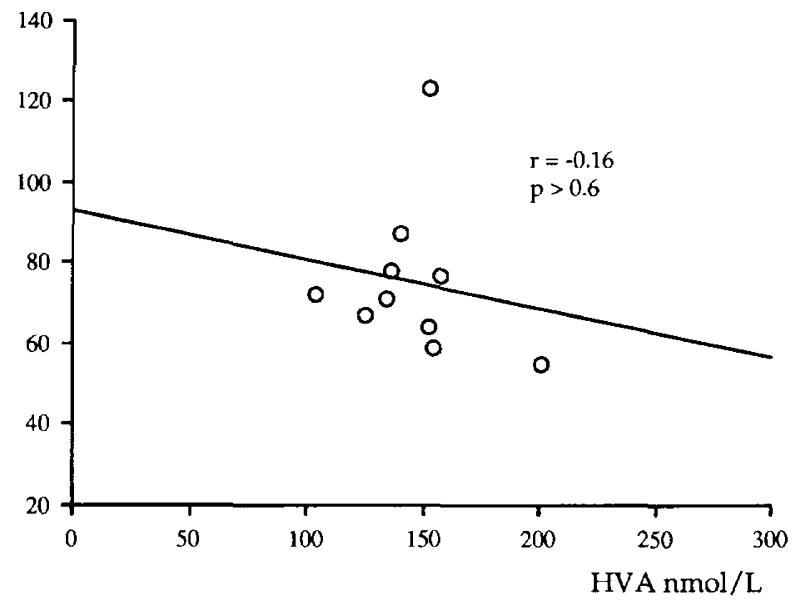

A
PMS: LUTEAL PHASE

5-HIAA nmol/L

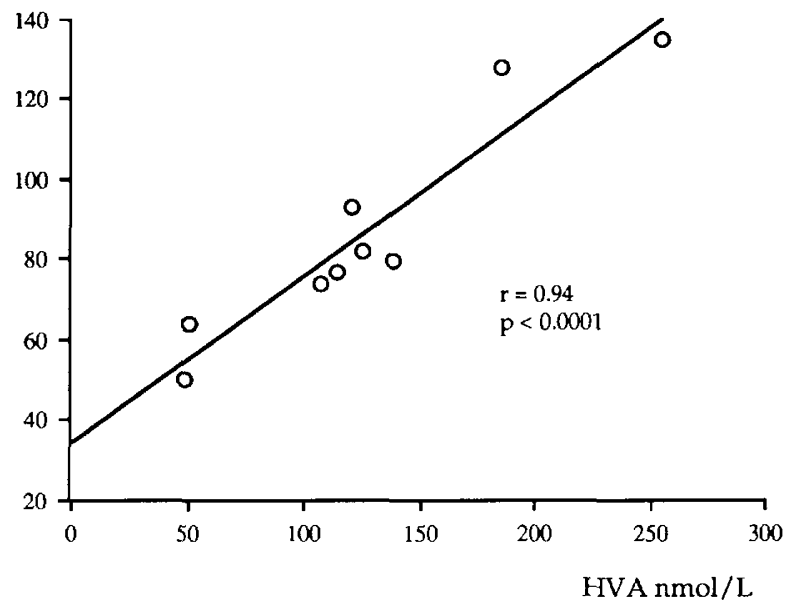

PMS: FOLLICULAR PHASE

5-HIAA nmol/L

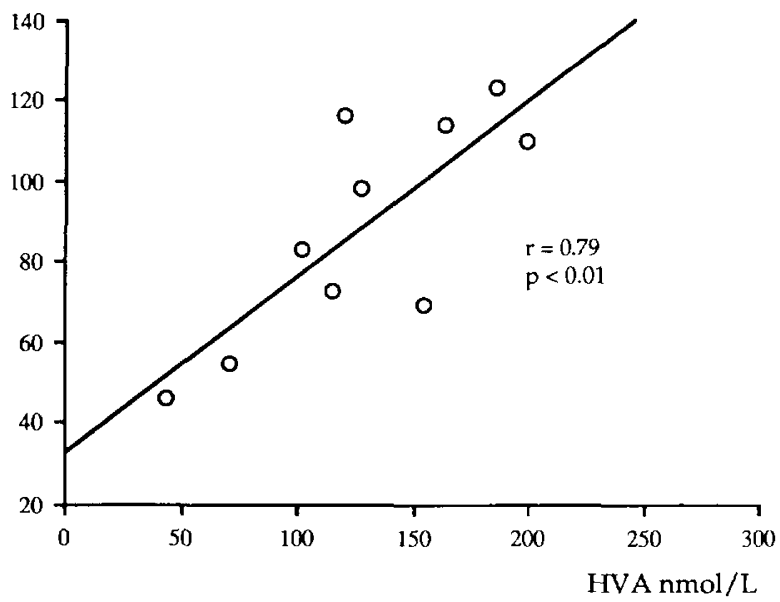

$\mathbf{B}$

Figure 3. Correlation between CSF 5-HIAA and CSF HVA in the luteal phase and follicular phase of the controls (A) and PMS patients (B).

$(p<.001)$ in the controls only. The HVA versus 5-HIAA correlation in the follicular phases was significantly stronger in PMS patients than in controls $(p<.01)$.

By means of ANOVA, a significant effect of diagnostic category $(F=9.4, p=.004)$, but not of phase $(F=1.3, p=.3)$, was observed with respect to the HVA to 5-HIAA ratio. The HVA to 5-HIAA ratio appeared lower in the PMS group both in the follicular phase and the luteal phase (Figure 4); however, the difference was statistically significant in the follicular phase only $(t=$ $2.8 ; p<.01)$. The 5 -HIAA to MHPG and HVA to MHPG ratios were not significantly influenced by cycle phase or diagnosis (data not shown).

CSF MHPG correlated weakly to CSF HVA (PMS, luteal phase: $r=0.46, p=.2$; PMS, follicular phase: $r=0.46, p=.2$; controls, luteal phase: $r=0.44, p=$ .2 ; controls, follicular phase $r=0.66 p=.04$ ) and CSF 5-HIAA (PMS, luteal phase: $r=0.44, p=.3$; PMS, follicular phase: $r=0.57, p=.08$; controls, luteal phase: $0.45, p=.2$; controls, follicular phase $r=0.22, p=.6$ ).

\section{Serum Concentrations of Sex Steroids and Pituitary Hormones}

Serum levels of estradiol, testosterone, progesterone, FSH, LH, SHBG, and CBG in patients and in controls are presented in Table 1. 
Table 1. Serum Concentrations of Pituitary Hormones and Sex Hormones in PMS Patients and in Controls

\begin{tabular}{|c|c|c|c|}
\hline & Controls & PMS & ANOVA \\
\hline Estradiol & & & $\mathrm{D}: p=0.09$ \\
\hline Follicular phase & $0.08 \pm 0.02$ & $0.32 \pm 0.12$ & Ph: $p=0.15$ \\
\hline Luteal phase & $0.30 \pm 0.06$ & $0.30 \pm 0.05$ & D*Ph: $p=0.09$ \\
\hline Progesterone & & & $\mathrm{D}: p=0.62$ \\
\hline Follicular phase & $1.72 \pm 0.26$ & $1.58 \pm 0.23$ & Ph: $p<0.0001$ \\
\hline Luteal phase & $26.21 \pm 6.49$ & $22.09 \pm 5.37$ & $\mathrm{D}^{*} \mathrm{Ph}: p=0.64$ \\
\hline Testosterone & & & $\mathrm{D}: p=0.003$ \\
\hline Follicular phase & $0.66 \pm 0.16$ & $1.14 \pm 0.16$ & Ph: $p=0.43$ \\
\hline Luteal phase & $0.70 \pm 0.13$ & $1.39 \pm 0.27$ & $\mathrm{D}^{*} \mathrm{Ph}: p=0.56$ \\
\hline SHBG & & & $\mathrm{D}: p=0.72$ \\
\hline Follicular phase & $50.46 \pm 5.70$ & $47.73 \pm 5.32$ & Ph: $p=0.77$ \\
\hline Luteal phase & $50.85 \pm 3.52$ & $50.14 \pm 3.83$ & $\mathrm{D} * \mathrm{Ph}: p=0.83$ \\
\hline CBG & & & $\mathrm{D}: p=0.09$ \\
\hline Follicular phase & $40.48 \pm 1.74$ & $38.79 \pm 1.95$ & Ph: $p=0.93$ \\
\hline Luteal phase & $43.22 \pm 2.29$ & $36.46 \pm 3.54$ & $D^{*}$ Ph: $p=0.31$ \\
\hline FSH & & & $\mathrm{D}: p=0.39$ \\
\hline Follicular phase & $1.65 \pm 0.11$ & $1.59 \pm 0.14$ & Ph: $p=0.003$ \\
\hline Luteal phase & $1.00 \pm 0.18$ & $1.31 \pm 0.13$ & $\mathrm{D}^{*} \mathrm{Ph}: p=0.20$ \\
\hline $\mathrm{LH}$ & & & $\mathrm{D}: p=0.44$ \\
\hline Follicular phase & $1.24 \pm 0.15$ & $1.37 \pm 0.29$ & Ph: $p=0.38$ \\
\hline Luteal phase & $1.01 \pm 0.21$ & $1.21 \pm 0.22$ & $\mathrm{D}^{*} \mathrm{Ph}: p=0.85$ \\
\hline Prolactin & & & $\mathrm{D}: p=0.01$ \\
\hline Follicular phase & $23.9 \pm 5.05$ & $13.01 \pm 6.95$ & Ph: $p=0.28$ \\
\hline Luteal phase & $26.22 \pm 3.37$ & $17.94 \pm 5.72$ & $\mathrm{D}^{*} \mathrm{Ph}: p=0.77$ \\
\hline
\end{tabular}

Units: estradiol, progesterone, testosterone, SHBG: nmol/L; transcortin: mg/L; prolactin, FSH, LH: $\mu \mathrm{g} / \mathrm{L}$. Abbreviations: $\mathrm{D}=$ diagnosis, $\mathrm{Ph}=$ cycle phase.

For testosterone, ANOVA revealed a significant influence of diagnostic group (levels being higher in PMS subjects than in controls; $p=.003$ ), but not of cycle phase. Subsequent 2 -tailed $t$-test disclosed significantly higher levels of testosterone in PMS subjects as compared to controls in the luteal phase $(p<.02)$ whereas the difference in the follicular phase did not reach statistical significance.
For prolactin, ANOVA revealed a significant influence of diagnostic group (levels being lower in PMS subjects than in controls; $p=.01$ ), but not of cycle phase. Subsequent 2-tailed $t$-test disclosed significantly lower levels of prolactin in PMS subjects as compared to controls in the follicular phase $(p<.05)$ whereas the difference in the luteal phase did not reach statistical significance.

Table 2. Correlations between CSF Monoamine Metabolites and Serum Hormone Concentrations in PMS Patients and in Controls

\begin{tabular}{|c|c|c|c|c|c|c|}
\hline & \multicolumn{2}{|c|}{ All Subjects } & \multicolumn{2}{|c|}{ PMS } & \multicolumn{2}{|c|}{ Controls } \\
\hline & Follicular Phase & Luteal Phase & Follicular Phase & Luteal Phase & Follicular Phase & Luteal Phase \\
\hline $\begin{array}{l}\text { MHPG vs. } \\
\text { LH }\end{array}$ & $\begin{array}{l}r=0.68 \\
p=0.001\end{array}$ & $\begin{array}{l}r=0.13 \\
p=0.60\end{array}$ & $\begin{array}{l}r=0.83 \\
p=0.004\end{array}$ & $\begin{array}{l}r=0.31 \\
p=0.4\end{array}$ & $\begin{array}{l}r=0.4 \\
p=0.3\end{array}$ & $\begin{array}{l}r=-0.14 \\
p=0.7\end{array}$ \\
\hline $\begin{array}{l}\text { MHPG } \\
\text { vs. test }\end{array}$ & $\begin{array}{l}r=0.12 \\
p=0.6\end{array}$ & $\begin{array}{l}r=-0.12 \\
p=0.6\end{array}$ & $\begin{array}{l}r=0.19 \\
p=0.6\end{array}$ & $\begin{array}{l}r=-0.80 \\
p=0.008\end{array}$ & $\begin{array}{l}r=0.09 \\
p=0.8\end{array}$ & $\begin{array}{l}r=0.48 \\
p=0.2\end{array}$ \\
\hline $\begin{array}{l}\text { MHPG } \\
\text { vs. estrogen }\end{array}$ & $\begin{array}{l}r=0.45 \\
p=0.05\end{array}$ & $\begin{array}{l}r=-0.23 \\
p=0.9\end{array}$ & $\begin{array}{l}r=0.72 \\
p=0.02\end{array}$ & $\begin{array}{l}r=-0.32 \\
p=0.4\end{array}$ & $\begin{array}{l}r=-0.55 \\
p=0.1\end{array}$ & $\begin{array}{l}r=0.21 \\
p=0.57\end{array}$ \\
\hline $\begin{array}{l}\text { MHPG } \\
\text { vs. prolactin }\end{array}$ & $\begin{array}{l}r=0.25 \\
p=0.3\end{array}$ & $\begin{array}{l}r=0.35 \\
p=0.1\end{array}$ & $\begin{array}{l}r=0.5 \\
p=0.1\end{array}$ & $\begin{array}{l}r=0.3 \\
p=0.4\end{array}$ & $\begin{array}{l}r=0.20 \\
p=0.6\end{array}$ & $\begin{array}{l}r=0.76 \\
p=0.009\end{array}$ \\
\hline $\begin{array}{l}\text { MHPG } \\
\text { vs. SHBG }\end{array}$ & $\begin{array}{l}r=-0.32 \\
p=0.18\end{array}$ & $\begin{array}{l}r=0.51 \\
p=0.02\end{array}$ & $\begin{array}{l}r=-0.1 \\
p=1.0\end{array}$ & $\begin{array}{l}r=0.76 \\
p=0.02\end{array}$ & $\begin{array}{l}r=-0.67 \\
p=0.03\end{array}$ & $\begin{array}{l}r=0.34 \\
p=0.34\end{array}$ \\
\hline $\begin{array}{l}\text { HVA } \\
\text { vs. LH }\end{array}$ & $\begin{array}{l}r=0.35 \\
p=0.1\end{array}$ & $\begin{aligned} r & =-0.05 \\
p & =0.8\end{aligned}$ & $\begin{array}{l}r=0.29 \\
p=0.5\end{array}$ & $\begin{array}{l}r=-0.08 \\
p=0.8\end{array}$ & $\begin{array}{l}r=0.72 \\
p=0.02\end{array}$ & $\begin{array}{l}r=0.02 \\
p=1.0\end{array}$ \\
\hline
\end{tabular}

Shown only are correlations with an $r$-value exceeding 0.7 for at least one cycle phase in at least one of the diagnostic groups. 


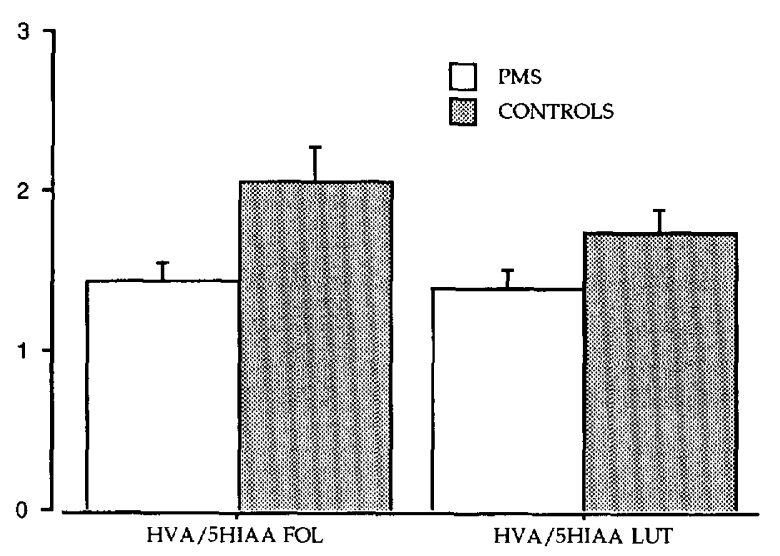

Figure 4. CSF HVA to 5-HIAA ratio in the follicular (FOL) phase and luteal (LUT) phase of PMS patients and controls. For statistics, see Results.

Serum ESTRADIOL $\mathrm{nmol} / \mathrm{L}$

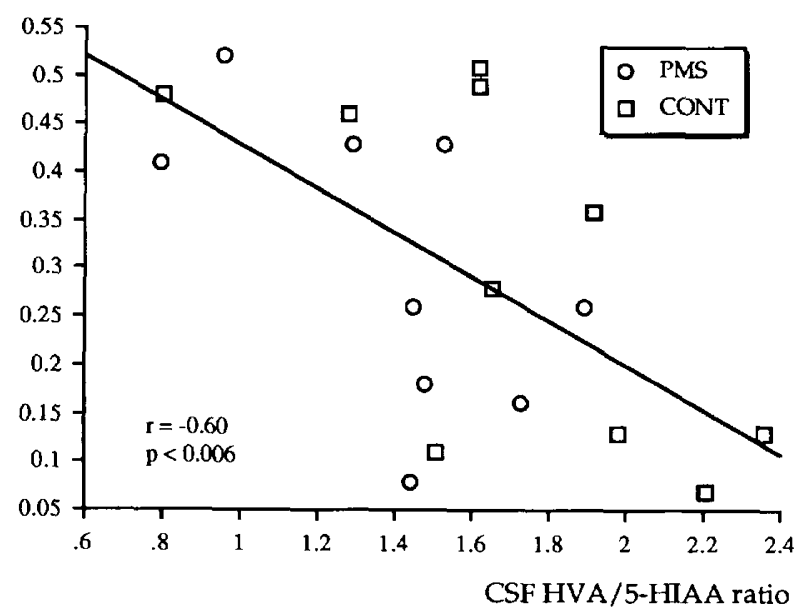

A

Serum TESTOSTERONE nmol/L

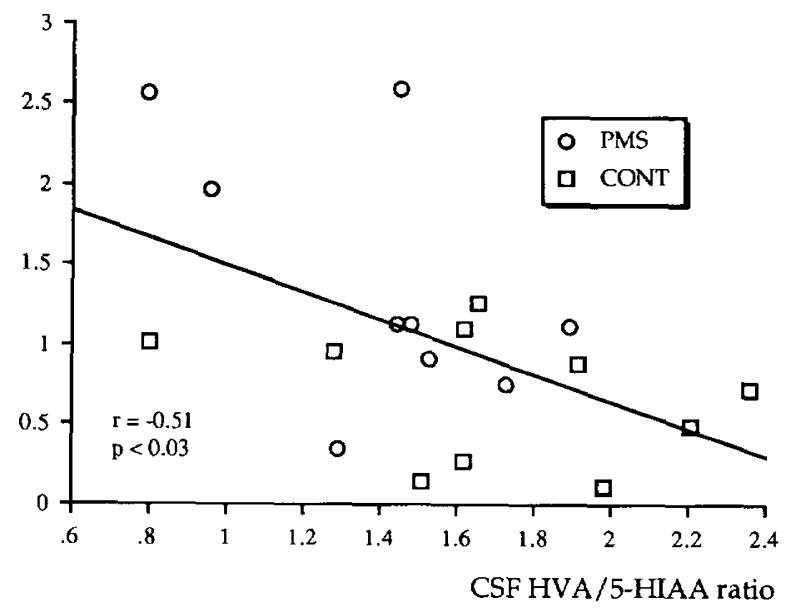

\section{Correlation between CSF Monoamine Metabolites and Hormones}

In Table 2 are shown all correlations between CSF monoamine metabolites versus sex steroids and pituitary hormones with $r$ greater than 0.7 in at least one cycle phase, in PMS subjects or in controls.

Given the large number of correlations, analyses undertaken, relations between two factors yielding $r<$ 0.7 were regarded as nonsignificant; these data are generally not shown. As an exception, a few correlations not exceeding an $r$ of 0.7 , but still regarded as being of possible physiological interest are presented in the text (see the following) and in Figures 5A-C, 6, and 7.

In the luteal phase, but not in the follicular phase, the CSF HVA to 5-HIAA ratio correlated negatively with

Serum PROGESTERONE nmol/L

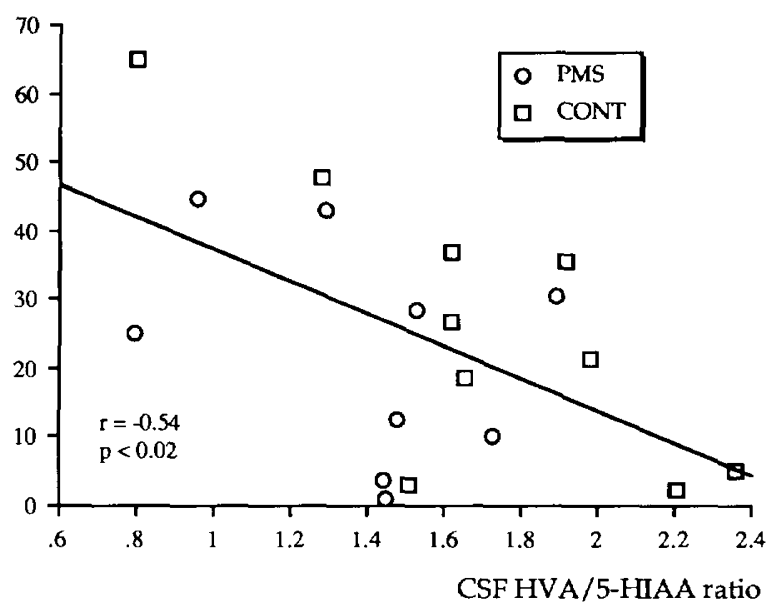

B

Figure 5. Correlation between serum estradiol (A), progesterone (B), and testosterone (C), respectively, and the CSF HVA to 5-HIAA ratio, in the luteal phase of PMS patients and controls. 
Serum $\mathrm{LH} \mu \mathrm{g} / \mathrm{L}$

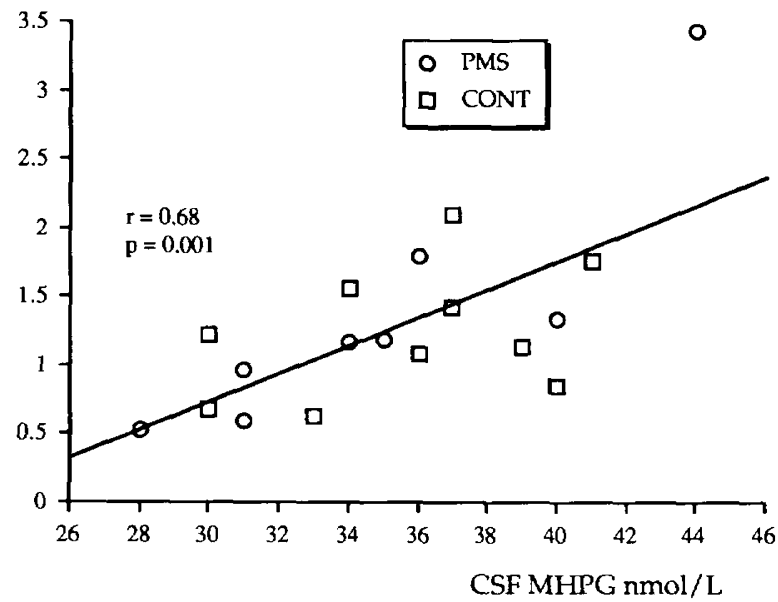

Figure 6. Correlation between serum LH and CSF MHPG concentration in the follicular phase of PMS patients and controls.

serum levels of estradiol, progesterone, and testosterone. In this respect, no significant difference between patients and controls was observed (Figure 5A-C).

In the entire group investigated, serum levels of $\mathrm{LH}$ correlated positively with CSF levels of MHPG in the follicular phase, but not in the luteal phase (Table 2, Figure 6).

In the entire group investigated, serum levels of prolactin correlated positively with CSF levels of HVA in the luteal phase, but not in the follicular phase (PMS, luteal phase: $r=0.68, p=.04$; PMS, follicular phase: $r=0.21, p=.6$; controls, luteal phase: $r=0.52, p=$ .1; controls, follicular phase: $r=0.09, p=.8$; also see Figure 7).

Serum PROLACTIN nmol/L

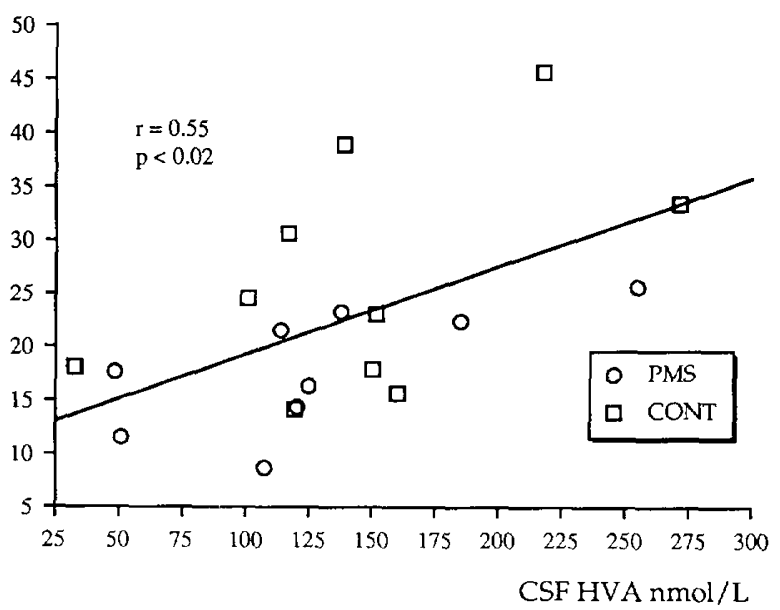

Figure 7. Correlation between serum prolactin and the CSF HVA concentration in the luteal phase of PMS patients and controls.

\section{DISCUSSION}

\section{CSF Monoamine Metabolites in Healthy Controls}

In the eight women without premenstrual complaints from which CSF samples were obtained both in the luteal phase and in the follicular phase, the intraindividual, intersample variations were found to be considerable for all three monoamine metabolites; in contrast, mean CSF metabolite concentrations in the follicular phase did not differ from those in the luteal phase.

Previous data on the intraindividual stability of CSF monoamine metabolites are sparse. Hildebrand and coworkers (1990), taking repeated samples from hospitalized patients with cognitive disorder, showed substantial intraindividual variations, but showed very small differences in group means with respect to CSF HVA, MHPG, and 5-HIAA. In a study by Träskman-Bendz and coworkers (1984), the variability in CSF samples obtained from healthy volunteers (male and female), with an interval of 2 weeks to 8 weeks, was relatively modest, with the correlations between the first value and the second value being stronger for all three metabolites than the correlations observed in the present study. The latter finding suggests that the large variability in CSF metabolite concentrations in controls observed in the present study may be because the samples were taken at different phases of the menstrual cycle rather than randomly with respect to cycle phase. Interestingly, in the study by Träskman-Bendz and coworkers (1984), larger intraindividual variations in CSF levels of 5-HIAA and HVA were found in females than in males.

Although the mean concentration of HVA in CSF were similar in the luteal and follicular phases, in normal controls the interindividual variation was considerably smaller in the follicular phase than in the luteal phase. Moreover, whereas in the luteal phase, the CSF concentrations of HVA and 5-HIAA strongly correlated, in the follicular phase, no such correlation was observed. This latter finding contrasts sharply to the large body of previous data showing a significant correlation between the serotonin and dopamine metabolites in various populations regardless of age, sex, and psychiatric diagnosis (Agren et al. 1986). However, albeit CSF concentrations of HVA and 5-HIAA have previously been frequently studied in women of fertile age, this is, to our knowledge, the first report on CSF monoamine concentrations obtained in the follicular phase before the midcycle rise in serum estradiol and progesterone. Hence, the lack of correlation between HVA and 5-HIAA in the early follicular phase is neither supported nor contradicted by earlier studies.

The possible differences in HVA variability, and in HVA vs 5-HIAA correlation, between samples obtained from the follicular and luteal phases, respectively, in- 
dicates that sex steroids may influence the dopamine activity and/or metabolism (cf. Di Paolo et al. 1989) as well as the interplay between dopamine and serotonin. Further support for this concept was obtained by the negative correlations between sex steroids and the HVA to 5-HIAA ratio observed in the luteal phase of the entire population (vide infra). Notably, in a study by Koslow and coworkers (1983), the HVA vs 5-HIAA correlation was stronger in female than in male healthy controls.

Due to the small number of subjects included in this study, the possibility that the small HVA variability, as well as the lack of HVA to 5-HIAA correlation, in the follicular phase of controls is accidental, should not be ignored.

\section{CSF Monoamine Metabolites in Women with PMS}

No difference was observed when PMS subjects and controls were compared with respect to mean concentrations of CSF 5-HIAA, HVA, and MHPG; moreover, like controls, women with PMS displayed no cycle related changes in mean CSF monoamine metabolite concentrations. The finding that CSF HVA and 5-HIAA levels in women with PMS are similar in the luteal and follicular phases is in agreement with a previous report from Parry and coworkers (1991). Our finding that MHPG levels are similar in the luteal and follicular phases is, however, in contrast with the study by Parry and coworkers which showed increased MHPG levels in the luteal phase in women with PMS.

In previous literature, a relation between low CSF 5-HIAA levels and proneness to respond to serotonin reuptake inhibitors have been suggested (Asberg et al., 1986a). However, given the dramatic therapeutic effect of serotonin reuptake inhibitors in the treatment of PMS (Eriksson et al. 1990; Rickels et al. 1990; Stone et al. 1991; Menkes et al. 1992; Sunblad et al. 1992; Wood et al. 1992; Sunblad et al. 1993), the present finding that CSF 5-HIAA levels appear normal in a group of women with PMS, when compared to controls with no premenstrual complaints, underlines that a low CSF level of 5-HIAA is not a characteristic feature of all conditions responding to antidepressants with a serotonergic profile. Similarly, previous studies have shown that CSF concentrations of 5-HIAA are not decreased in patients with panic disorder (Eriksson et al. 1992) or obsessive compulsive disorder (Thorén et al. 1980; Insel et al. 1985) in spite of the fact that these conditions also respond favorably to serotonin reuptake inhibitors (Eriksson and Humble 1990).

The finding that CSF 5-HIAA levels are not lower in PMS subjects as compared to controls, and also not lower in the luteal phase as compared to the follicular phase, does not exclude the possibility that PMS is associated with a sex steroid induced change in serotonin turnover in a restricted region of the brain not reflected by the CSF 5-HIAA concentration. Also, the possibility that PMS may be associated with a change in serotonin receptor responsiveness - rather than in serotonin metabolism-should not be ignored (cf Yatham et al. 1989). Several earlier studies do lend support for the concept that premenstrual changes in mood are indeed associated with measurable changes in various aspects of serotonergic function in the brain and in the periphery (Taylor et al. 1984; Ashby et al. 1988; Bancroft et al. 1991; Rojansky et al. 1991).

Although mean concentrations of CSF monoamine metabolites did not separate patients from controls, the intraindividual variability, when comparing samples from the follicular phase and the luteal phase, respectively, was significantly lower in PMS subjects than in controls for both HVA and 5-HIAA. As in the controls, the correlation between HVA and 5-HIAA appeared weaker in the follicular phase as compared to the luteal phase also in women with PMS; however, the HVA vs 5-HIAA correlation in the follicular phase was significantly higher in PMS subjects than in the controls, and the difference between the two phases with respect to HVA vs 5-HIAA correlation reached statistical significance in the controls only. Thus, with respect to individual HVA and 5-HIAA levels, as well as the HVA vs 5-HIAA correlation, PMS patients appeared less influenced by cycle phase than did the controls. When interpreting this apparent difference between patients and the controls, it should be taken into consideration that the serum concentrations of estradiol in the follicular phase tended to be higher in women with PMS than in the controls ( $p=.06)$; thus, information regarding CSF monoamine concentrations in a situation with very low $(<0.1 \mathrm{nmol} / \mathrm{L})$ serum levels of estradiol was obtained from the controls only.

Several recent papers have emphasized the possible importance of the HVA to 5-HIAA ratio as an index of serotonin/dopamine interactions and as a marker of psychiatric morbidity (Ågren et al., 1986; Roy et al., 1986; Risby et al. 1987; Agren et al. 1988; Hsiao et al. 1993; Potter and Manji, 1993). Interestingly, in depression, a reduced HVA to 5-HIAA ratio appears to be a more robust biological marker than the actual concentrations of HVA and 5-HIAA, respectively (Roy et al. 1986; Reddy et al. 1992). Hence, the present finding that the HVA to 5-HIAA ratio may be lower in women with PMS than in the controls suggests that PMS and depression may have pathophysiological mechanisms in common; needless to say, the efficacy of serotonin reuptake inhibitors in the treatment of PMS (for references, see previous) lends further support for this concept.

\section{Pituitary and Sex Hormones in PMS Patients and Controls}

A comparison of PMS patients and controls with respect to serum hormonal levels was not the primary 
scope of this paper; thus, hormonal levels in a larger group, of which the women exposed to lumbar puncture constitute a subpopulation, will be presented elsewhere. However, it should be noted that serum levels of total testosterone were significantly higher in women with PMS as compared to controls; this finding is in line with our previously reported observation, based on data from different groups of PMS patients and controls, showing significantly higher levels of free testosterone in women with PMS (Eriksson et al. 1992). Also noteworthy is our finding that serum levels of prolactin were significantly lower in the follicular phase of PMS subjects (cf Andersch et al. 1979).

\section{Influence of Sex Steroids on the HVA to 5-HIAA Ratio}

When all subjects-patients and controls-were analyzed together, the HVA vs 5-HIAA correlation turned out to be considerably stronger in the luteal phase, when serum levels of sex steroids are high, than in the follicular phase, when estradiol and progesterone are low $(p<.01)$; hence, it may be suggested that the interaction between HVA and 5-HIAA in women could be under the influence of steroid hormones. In support for this assumption, in the luteal phase the HVA to 5-HIAA ratio (but neither the HVA nor the 5-HIAA concentration per se) correlated negatively with estradiol, progesterone, and total testosterone.

The mechanisms underlying the strong correlation between CSF HVA and 5-HIAA usually observed in man is obscure (for discussion, see Agren et al. 1986; Hsiao et al. 1993), and so is the possible influence of sex steroids on this interaction. According to one hypothesis, CSF 5-HIAA concentrations is a reflection of the monoamine oxidase (MAO) activity rather than of serotonin synthesis or release (Wolf et al. 1985). Because in man both serotonin and dopamine are metabolized by MAO A and MAO B, the possibility that a change in MAO activity may change the relation between 5-HIAA and HVA in CSF should not be ignored. In this context, the well established influence of estradiol, testosterone, and progesterone on brain MAO A and MAO B activity (Luine et al. 1975; Leung et al. 1980; Chevillard et al. 1981; Vaccari and Biassoni 1982; Luine and Rhodes 1983) is worth recalling; however, the physiological significance of this steroid influence on MAO activity still remains to be established. Moreover, animal experiments have revealed multiple examples of interactions between serotonin and dopamine not related to MAO activity (e.g. Ågren et al. 1986; Benloucif and Galloway 1991; Nissbrandt et al. 1992); likewise, sex steroids have been shown to influence both dopamine and serotonin activity by a number of various mechanisms (Engel et al. 1979; Sietniks et al. 1983; Long et al. 1983; Battaner et al. 1987; Bitar et al. 1991). Obviously, further studies are warranted in order to clarify the possible relations between sex steroids and the CSF HVA to 5-HIAA ratio.

\section{Relation between Pituitary Hormones and CSF Monoamine Metabolites}

Because a large number of correlation analyses were undertaken when evaluating the possible interplay between CSF monoamine metabolites on the one hand and gonadal and pituitary hormones on the other, correlations of weak significance and without obvious physiological significance should be interpreted with caution; however, we observed two examples of an apparent covariance between a pituitary hormone and a CSF monoamine metabolite that deserves attention.

First, in the follicular phase, serum levels of LH correlated strongly to CSF levels of the noradrenaline metabolite, MHPG. Earlier animal studies have shown noradrenaline to be a major regulator of $\mathrm{GnRH} / \mathrm{LH}$ release; thus, the progesterone induced midcycle $\mathrm{LH}$ surge appears to be noradrenaline mediated (see Adler et al. 1983). When interpreting the observed correlation between LH and MHPG, both central and peripheral mechanisms should be considered. Thus, it has been suggested that CSF MHPG partly emanates from peripheral sources (Kopin et al. 1983); moreover, several studies have shown that the midcycle LH surge is accompanied by an increase in serum levels of noradrenaline (Badano et al. 1978; also see Nagle and Rosner 1980). Interestingly, in a recent study in women with polycystic ovary syndrome, plasma MHPG levels were shown to correlate with the magnitude of the LH response to LHRH, indicating that noradrenaline influences the responsiveness of the pituitary to the LH releasing hormone (Yoshino et al. 1992).

Second, in the luteal phase, but not in the follicular phase, serum levels of prolactin correlated positively with CSF levels of HVA. Whereas tuberoinfundibular dopamine neurons inhibits prolactin release, prolactin has been shown to stimulate dopamine activity in the hypothalamus (Hökfelt and Fuxe 1972), and, perhaps, also in the striatum (Bédard et al. 1984). Further studies are warranted to establish whether the observed correlation between CSF HVA and serum prolactin is a reflection of the physiological interplay between dopamine and prolactin, or merely accidental.

\section{CONCLUSION}

In a small group of healthy women without premenstrual complaints, the interindividual variability in CSF HVA levels appeared smaller, and the HVA vs 5-HIAA correlation weaker, in the follicular phase than in the luteal phase; in contrast, the menstrual cycle phase did not influence the mean concentrations of CSF mono- 
amine metabolites. In PMS patients, the intraindividual variability of CSF metabolites seemed smaller than in controls; moreover, in the follicular phase, the interindividual HVA variability appeared greater, the HVA vs 5-HIAA correlation stronger, and the HVA to 5-HIAA ratio lower than in controls. In the entire group of subjects investigated, the CSF HVA to 5-HIAA ratio correlated negatively with serum levels of estradiol, progesterone, and testosterone in the follicular phase. Albeit these findings were obtained from small groups of subjects and hence should be interpreted with great caution, they may encourage further studies on the possible influence of sex steroids on CSF concentrations of HVA and 5-HIAA and on the relation between these two metabolites. When CSF samples are obtained for determination of monoamine metabolites from women of fertile age, the menstrual cycle phase at the time of sampling is a factor of a putative importance that should not be ignored.

\section{ACKNOWLEDGMENT}

This study was supported by grants from the Swedish Medical Research Council (04752 and 08668). Excellent technical assistance was provided by Pia Lisjö and Birgitta Holmberg. Professor Hans Agren is gratefully acknowledged for valuable discussions.

\section{REFERENCES}

Adler BA, Johnson MD, Lynch CO, Crowley WR (1983): Evidence that norepinephrine and epinephrine systems mediate the stimulatory effects of ovarian hormones on luteinizing hormone and luteinizing hormone-releasing hormone. Endocrinology 113:1431-1438

Ågren H, Mefford IN, Rudorfer MV, Linnoila M, Potter WZ (1986): Interacting neurotransmitter systems. A nonexperimental approach to the 5-HIAA-HVA correlation in human CSF. J Psychiatr Res 20:175-193

Ågren H, Nordin C, Potter WZ (1988): Antidepressant drug action and cerebrospinal fluid monoamine metabolites: New evidence for selective profiles on monoaminergic interactions. In: Progress in Catecholamine Research, Part C: Clinical Aspects. Alan R. Liss, Inc, pp 307-311

American Psychiatry Association (1985): Diagnostic and statistical manual of mental disorders, ed 3, revised version. Washington DC, AP Press, pp 367-369

Andersch B, Abrahamsson L, Wendestam C, Öhman R, Hahn $\mathrm{L}$ (1979): Hormone profile in the premenstrual tension: Effects of bromocriptine and diuretics. Clin Endocrinol 11:657-664

Åsberg M, Bertilsson L, Mărtensson B, Scalia-Tomba G-P, Thorén P, Träskman-Bendtz L (1984): CSF monoamine metabolites in melancholia. Acta Psych Scand 69:201-219

Åsberg M, Eriksson B, Mårtensson B, Träskman-Bendz L, Wagner A (1986a): Therapeutic effects of serotonin reuptake inhibitors in depression. J Clin Psychiatry 47:23-35
Åsberg M, Nordström P, Träskman-Bendz L (1986b): Cerebrospinal fluid studies in suicide. An overview. Ann N Y Acad Sci 487:243-255

Ashby Jr CR, Carr LA, Cook CL, Steptoe MM, Franks DD (1988): Alterations of platelet serotonergic mechanisms and monoamine oxidase activity in premenstrual syndrome. Biol Psychiatry 24:225-233

Badano AR, Nagle CA, Figueroa Casas PR, Miechi H, Mirkin A, Turner DE, Aparicio N, Rosner JM (1978): Plasma levels of norepinephrine during the periovulatory period in normal women. Am J Obstet Gynecol 131:299-303

Bancroft J, Cook A, Davidson D, Bennie J, Goodwin G (1991): Blunting of neuroendocrine responses to infusion of L-tryptophan in women with perimenstrual mood change. Psychological Med 21:205-312

Battaner E, Rodriguez-del-Castillo A, Guerra M, Mas M (1987): Gonadal influences on spinal cord and brain monoamines in male rats. Brain Res 10:391-394

Bédard P, Boucher R, Di Paolo T, Labrie F (1984): Interaction between estradiol, prolactin, and striatal dopaminergic mechanisms. Adv Neurol 40:489-495

Benloucif S, Galloway M (1991): Facilitation of dopamine release in vitro by serotonin agonists: studies with microdialysis. Eur J Pharmacology 200:1-8

Bitar MS, Ota M, Linnoila M, Shapiro BH (1991): Modification of gonadectomy-induced increases in brain monoamine metabolism by steroid hormones in male and female rats. Psychoneuroendocrinology 16:547-557

Brown GL, Ebert M, Goyer P, Jimerson D, Klein WJ, Bunney W, Goodwin F (1982): Aggression, suicide and serotonin: Relationships to CSF amine metabolites. Am J Psychiatry 139:741-746

Brown GL, Linnoila M (1990): CSF serotonin metabolite (5-HIAA) studies in depression, impulsivity and violence. J Clin Psychiatry 51:31-41

Chevillard C, Barden N, and Saavedra JM (1981): Estradiol treatment decreases type $A$ and increases type $B$ monoamine oxidase in specific brain stem areas and cerebellum of ovariectomized rats. Brain Res 222:177-181

Di Paolo T, Bédard F, Bédard PJ (1989): Influence of gonadal steroids on human and monkey cerebrospinal fluid homovanillic acid concentrations. Clin Neuropharmacol 12:60-66

Engel J, Ahlenius S, Almgren O, Carlsson A, Larsson K, Södersten P (1979): Effects of gonadectomy and hormone replacement on brain monoamine synthesis in male rats. Pharmacol Biochem Behav 10:149-154

Eriksson E, Lisjö P, Sundblad C, Andersson K, Andersch B, Modigh K (1990): Effects of clomipramine on premenstrual syndrome. Acta Psychiatr Scand 81:87

Eriksson E, Humble M (1990): Serotonin in psychiatric pathophysiology. A review of data from experimental and clinical research. In Gershon S, Pohl R (eds), Prog Basic Clin Pharmacol, Vol 3, Biological Basis of Psychiatric Treatment. Basel, Karger, pp 66-119

Eriksson E, Sundblad C, Lisjö P, Modigh K, Andersch B (1992): Serum levels of androgens are higher in women with premenstrual irritability and dysphoria than in controls. Psychoneuroendocrinology 17:195-204

Eriksson E, Westberg P, Alling C, Thuresson K, Modigh K 
(1991): Cerebrospinal fluid levels of monoamine metabolites in panic disorder. Psychiatry Res 36:243-251

Frank RT (1931): The hormonal causes of premenstrual tension. Arch Neurol Psychiatry 26:1053-1057

Gibbons RD, Davis JM (1984): Consistent evidence for a biological subtype of depression characterized by low CSF monoamine metabolite levels. Acta Psychiatr Scand $74: 8-12$

Gjerris A (1988): Baseline studies on transmitter substances in cerebrospinal fluid in depression. Acta Psychiatr Scand 78:1-35

Gjerris A, Werdelin L, Gjerris F, Sorensen PS, Rafaelsen OJ, Alling C (1987): CSF-amine metabolites in depression, dementia and controls 75:619-628

Halbreich U, Endicott J, Schnacht S, Nee J (1982): The diversity of premenstrual changes as reflected in the Premenstrual Assessment Form. Acta Psychiatr Scand 65:46-65

Hildebrand J, Bourgeois F, Buyse M, Brzedborski S, Goldman S (1990): Reproducibility of monoamine metabolite measurements in human cerebrospinal fluid. Acta Neurol Scand 81:427-430

Hökfelt T, Fuxe K (1972): Effects of prolactin and ergot alkaloids on the tuberoinfundibular dopamine (DA) neurons. Neuroendocrinology 9:100-122

Hsiao JK, Potter WZ, Ågren H, Owen RR, Pickar D (1993): Clinical investigation of monoamine neurotransmitter interactions. Psychopharmacology 112 (Suppl 1):S76-S84

Insel TR, Mueller EA, Alterman I, Linnoila M, Murphy DL (1985): Obsessive-compulsive disorder and serotonin: Is there a connection? Biol Psychiatry 20:1174-1188

Kopin IJ, Gordon EK, Jimerson DC, Polinsky RJ (1983): Relationship between plasma and cerebrospinal fluid levels of 3-methoxy-4-hydroxyphenylglycol. Science 219:73-75

Koslow SH, Maas JW, Bowden CL, Davis JM, Hanin I, Javaid J (1983): CSF and urinary biogenic amines and metabolites in depression and mania. Arch Gen Psychiatry 40:999-1010

Leung TK, Lai JC, Marr W, Lim L (1980): The activities of the $A$ and $B$ forms of monoamine oxidase in liver, hypothalamus and cerebral cortex of the female rat: Effects of administration of ethinylestradiol and the progestogens norethisterone acetate and d-norgestrel. Biochem Soc Trans 8:607-608

Lidberg L, Tuck JR, Åsberg M, Scalia-Tomba G-P, Bertilsson L (1985): Homicide, suicide and CSF 5-HIAA. Acta Psychiatr Scand 71:230-236

Long JB, Youngblood WW, Kizer JS (1983): Effects of castration and adrenalectomy on in vitro rates of tryptophan hydroxylation and levels of serotonin in microdissected brain nuclei of adult male rats. Brain Res 277:289-297

Luine VN, Khylchevskaya RI, McEwen BS (1975): Effect of gonadal steroids on activities of monoamine oxidase and choline acetylase in rat brain. Brain Res 86:293-306

Luine VN, Rhodes JC (1983): Gonadal regulation of MAO and other enzymes in hypothalamic areas. Neuroendocrinology 36:235-241

Menkes DB, Taghavi E, Mason PA, Spears GFS, Howard RC (1992): Fluoxetine treatment of severe premenstrual syndrome. BMJ 305:346-347
Modigh K (1975): The relationship between the concentrations of tryptophan and 5-hydroxyindoleacetic acid in rat brain and cerebrospinal fluid. I Neurochemistry 25: 351-352

Nagle CA, Rosner JM (1980): Rat brain norepinephrine release during progesterone-induced LH secretion. Neuroendocrinology 30:33-37

Nissbrandt H, Waters N, Hjorth S (1992): The influence of serotonergic drugs on dopaminergic neurotransmission. Naunyn-Schmiedeberg's Arch Pharmacol 346:12-19

Parry BL, Gerner RH, Wilkins JN, Halaris AE, Carlson HE, Hershman JM, Linnoila M, Merrill J, Gold PW, Gracely R, Aloi J, Newton R (1991): CSF and endocrine studies of premenstrual syndrome. Neuropsychopharmacology 5:127-137

Potter WZ, Manji HK (1993): Are monoamine metabolites in cerebrospinal fluid worth measuring? Arch Gen Psychiatry 50:653-656

Reddy PL, Khanna S, Subhash MN, Channabasavanna SM, Rama Rao BSS (1992): CSF amine metabolites in depression. Biol Psychiatry 31:112-118

Rickels K, Freedman EW, Sondheimer S, Albert J (1990): Fluoxetine in the treatment of premenstrual syndrome. Curr Ther Res 48:161-166

Risby ED, Hsiao JK, Sunderland T, Agren H, Rudorfer MV, Potter WZ (1987): The effects of antidepressants on the cerebrospinal fluid homovanillic acid/5-hydroxyindoleacetic acid ratio. Clin Pharmacol Ther 42:547-554

Rojansky N, Halbreich U, Zander K, Barkai A, Goldstein S (1991): Imipramine receptor binding and serotonin uptake in platelets of women with premenstrual changes. Gynecol Obstet Invest 31:146-152

Roy A, Ågren H, Pickar D, Linnoila M, Doran AR, Cutler NR, Paul SM (1986): Reduced CSF concentrations of homovanillic acid and homovanillic acid to 5-hydroxyindoleacetic acid ratios in depressed patients: Relationship to suicidal behavior and dexamethasone nonsuppression. Am J Psychiatry 143:1539-1545

Scheinin H, MacDonald E, Scheinin M (1986): Comparison of free MHPG in rat cerebrospinal fluid with free and conjugated MHPG in brain tissue: effects of drugs modifying noradrenergic transmission. European J Pharmacol 129:113-121

Sietnieks A, Andén N-E, Grabowska-Andén M (1983): Determination of serotonin in small brain structures by means of HPLC combined with electrochemical detection: The effect of ovarian steroids on serotonin turnover rate within several brain regions of the ovariectomized rat. Acta Pharmacol Toxicol 52:398-400

Stanley M, Stanley B, Träskman-Bendtz L, Mann JJ, Meyendorff E (1986): Neurochemical findings in suicide completers and suicide attempters. Suicide Life Threat Behav 16:286-300

Stone AB, Pearlstein TB, Brown WA (1991): Fluoxetine in the treatment of late luteal phase dysphoric disorder. J Clin Psychiatry 52:290-293

Sundblad C, Andersch B, Modigh K, Eriksson E (1992): Clomipramine effectively reduces premenstrual irritability and dysphoria: a placebo-controlled trial. Acta Psychiatr Scand 85:39-47 
Sundblad C, Hedberg MA, Eriksson E (1993): Clomipramine administered during the luteal phase reduces the symptoms of premenstrual syndrome: A placebo-controlled trial. Neuropsychopharmacology 9:133-145

Swahn CG, Sandgarde B, Wiesel F-A, Sedvall G (1976): Simultaneous determination of the three major monoamine metabolites in brain tissue and body fluids by a mass fragmentographic method. Psychopharmacology 48:147-153

Taylor DL, Mathew RJ, Ho BT, Weinman ML (1984): Serotonin levels and platelet uptake during premenstrual tension. Neuropsychobiology 12:16-18

Thorén P, Åsberg M, Bertilsson L, Mellström B, Sjöqvist F, Träskman L (1980): Clomipramine treatment of obsessive-compulsive disorder. II. Biochemical aspects. Arch Gen Psychiatry 37:1289-1294

Träskman-Bendz L, Åsberg M, Bertilsson L, Thorén P (1984): CSF monoamine metabolites of depressed patients during illness and after recovery. Acta Psychiatr Scand 69:333-342

Vaccari A, Biassoni R (1982): Gonadal influences on the inhi- bition of monoamine oxidase type B activity. J Neurosci Res 8:13-19

Westenberg HGM, Verhoeven WMA (1988): CSF monoamine metabolites in patients and controls: support for a bimodal distribution in major affective disorders. Acta Psychiatr Scand 78:541-549

Wood SH, Mortola JF, Chan YF, Moossazadeh F, Yen SS (1992): Treatment of premenstrual syndrome with fluoxetine: A double-blind, placebo-controlled, crossover study. Obstet Gynecol 80:339-344

Wolf WA, Youdim MBH, Kuhn DM (1985): Does brain 5-HIAA indicate serotonin release or monoamine oxidase activity? Eur J Pharmacology 109:381-387

Yatham LM, Barry S, Dinan TG (1989): Serotonin receptors, buspirone, and premenstrual syndrome. Lancet 8652 : 1447-1448

Yoshino K, Takahashi K, Nishigaki A, Eda Y, Kitao M (1992): Further evidence concerning catecholamine metabolism in polycystic ovary syndrome following $\mathrm{Gn}-\mathrm{RH}$ administration. Int J Fertil 37:111-114 